\title{
Cohorte histórica de artroplastia unicompartimental de rodilla en un hospital universitario chileno
}

\section{Historical Cohort of Unicompartmental Knee Arthroplasty in a Chilean University Hospital}

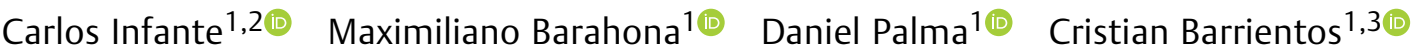

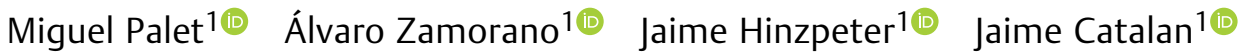 \\ ${ }^{1}$ Departamento de Ortopedia, Hospital Clínico Universidad de Chile,
Santiago, Chile
${ }^{2}$ Departamento de Ortopedia, Clínica las Condes, Santiago, Chile
${ }^{3}$ Departamento de Ortopedia, Clínica Santa María, Santiago, Chile \\ Address for correspondence Maximiliano Barahona, MD Biostat, \\ Santos Dumont 999, 3er piso, oficina 351, Independencia, código \\ postal 8380456, Santiago, Chile \\ (e-mail: maxbarahonavasquez@gmail.com).
}

Rev Chil Ortop Traumatol 2022;63(1):e25-e32.

\section{Resumen \\ Palabras Clave \\ - artroplastia unicompartimental de rodilla \\ - reemplazo de rodilla unicompartimental \\ - osteoartritis \\ - rodilla \\ - epidemiología}

Objetivo Describir el resultado funcional informado por el paciente de una cohorte de pacientes sometidos a artroplastia unicompartimental de rodilla (AUR) en un hospital universitario chileno.

Métodos Se diseñó un estudio de cohorte histórica. Se incluyeron todos los pacientes que se sometieron a AUR de platillo fijo entre 2003 y 2019. Un evaluador independiente se puso en contacto con los pacientes en junio de 2020. Se utilizó el índice de artritis de las universidades de Western Ontario y McMaster (WOMAC) para comparar los procedimientos de AUR (medial o lateral), la edad (mayor o menor de 70 años), y el seguimiento (más o menos de 5 años).

Resultados Se incluyeron 78 pacientes, en un total de 94 AURs. La mediana de edad fue de 64 años (rango: 43 a 85 años). Hubo 72 (76,6\%) casos de AUR medial. Un paciente necesitó revisión para artroplastia total de rodilla (ATR). Un total de 60 pacientes (76,9\%), correspondientes a 72 AURs (76,7\%), fueron contactados con éxito por teléfono para el seguimiento final. La mediana del puntaje en los dominios del WOMAC fue: dolor - 1 (rango: 0 a 12); rigidez - 0 (rango: 0 a 4); y función física - 2 (rango: 0 a 29). La mediana del puntaje total en el WOMAC fue de 4 (rango: 0 a 44). Los pacientes sometidos a AUR lateral lograron mejores puntuaciones funcionales $(p=0,0432)$, y el puntaje total en el WOMAC fue similar en pacientes mayores 0 menores de 70 años $(p=0,3706)$.

Conclusiones La AUR es un tratamiento eficaz y reproducible para pacientes con artrosis de rodilla unicompartimental. La edad parece no afectar los resultados recibido

28 de marcha de 2021

aceptado

06 de agosto de 2021

published online

January 17, 2022
DOI https://doi.org/ 10.1055/s-0041-1740641. ISSN 0716-4548.
(C) 2022. Sociedad Chilena de Ortopedia y Traumatologia. All rights reserved.

This is an open access article published by Thieme under the terms of the Creative Commons Attribution-NonDerivative-NonCommercial-License, permitting copying and reproduction so long as the original work is given appropriate credit. Contents may not be used for commercial purposes, or adapted, remixed, transformed or built upon. (https://creativecommons.org/ licenses/by-nc-nd/4.0/)

Thieme Revinter Publicações Ltda., Rua do Matoso 170, Rio de Janeiro, RJ, CEP 20270-135, Brazil 
funcionales, y la AUR es un tratamiento eficaz en pacientes mayores de 70 años. Estos resultados deberían animar a los cirujanos de rodilla a aprender esta técnica y a los responsables de las políticas de salud pública a considerar la AUR para la osteoartritis de rodilla.

\author{
Abstract \\ Keywords \\ - unicompartmental \\ knee arthroplasty \\ - unicompartmental \\ knee replacement \\ - osteoarthritis \\ - knee \\ - epidemiology
}

Purpose To describe the patient-reported functional outcome of a cohort of patients undergoing unicompartmental knee arthroplasty (UKA) in a Chilean university hospital.

Methods A historical cohort study was designed. All patients who underwent fixedbearing UKA between 2003 and 2019 were included. An independent evaluator contacted the patients in June 2020. The Western Ontario and McMaster Universities Arthritis Index (WOMAC) was used to compare UKA procedures (medial or lateral), age (over or under 70 years), and follow up (longer or shorter than 5 years). Results A total of 78 patients, corresponding to 94 UKAs, were included. The median age was 64 years (range: 43 to 85 years). There were $72(76.6 \%)$ cases of medial UKA. One patient needed revision to total knee arthoplasty (TKA). A total of 60 patients (76.9\%), corresponding to 72 UKAs, were successfully contacted by phone for the final follow-up. The median scores on the WOMAC domains were: pain - 1 (range: 0 to 12); stiffness -0 (range: 0 to 4 ); and physical function - 2 (range: 0 to 29). The median total score on the WOMAC was 4 (range: 0 to 44). Patients submitted to lateral UKA had better functional scores $(p=0.0432)$, and the total WOMAC score was similar among patients older or younger than 70 years of age $(p=0.3706)$.

Conclusions For patients with unicompartmental knee osteoarthritis, UKA is an effective and reproducible treatment. Age does not seem to affect the functional results, and UKA is an effective treatment in patients over 70 years old. These results should encourage knee surgeons to learn this technique and those responsible for public health policies to consider UKA for knee osteoarthritis.

\section{Introducción}

La artroplastia unicompartimental de rodilla (AUR) se introdujo por primera vez en la década de 1970 para pacientes con osteoartritis unicompartimental tibiofemoral grave. $^{1,2}$

La AUR es un tratamiento rentable en pacientes con osteoartritis del compartimento tibiofemoral medial o lateral, y logra de manera constante una alta tasa de resultados informados por los pacientes en la categoría de bueno/excelente. ${ }^{3}$ Además, se reporta una tasa de retorno al deporte más alta que la de la artroplastia total de rodilla (ATR). ${ }^{4}$ El registro de artroplastias del Reino Unido muestra que entre el $8 \%$ y el $15 \%$ de los pacientes que necesitan artroplastia de rodilla pueden ser tratados con éxito con una AUR. $^{5}$

Nuestra institución es un centro de salud universitario que pertenece a la red de atención privada chilena. A pesar de esto, alrededor de la mitad de nuestros pacientes pertenecen al seguro público de salud (Fondo Nacional de Salud, conocido como FONASA). La AUR en Chile no se ha convertido en una técnica ampliamente utilizada como la ATR. Desafortunadamente, no hay datos objetivos disponibles, pues el sistema de codificación nacional no diferencia entre AUR y ATR. Sin embargo, nuestro centro es uno de los que tiene mayor volumen de AUR en Chile. Se dan diferentes razones para esta menor cantidad de AUR, entre ellas: menor acceso al aprendizaje de la técnica, curva de aprendizaje más lenta, y mayor dificultad en comparación con la ATR. ${ }^{6}$ Sin embargo, la AUR ha mostrado buenos resultados en series internacionales tanto en pacientes jóvenes como geriátricos, ${ }^{7,8}$ por lo que es de gran interés seguir difundiendo este procedimiento quirúrgico para que se incorpore al arsenal de tratamiento de los pacientes con artrosis de rodilla en nuestro país. ${ }^{9}$

El propósito de este estudio es describir el resultado funcional informado por los pacientes de la cohorte histórica de pacientes sometidos a AUR en nuestro centro, y comparar los resultados con los informes internacionales. Presumimos que los resultados de nuestra cohorte son comparables con los de las series internacionales, independientemente del seguimiento, de la edad, y del lado de la prótesis.

\section{Métodos}

Se diseñó y realizó un estudio de cohorte histórica en el Hospital Clínico Universidad de Chile. Los pacientes que se 
someten a artroplastia de rodilla en nuestro centro están registrados según el sistema de codificación nacional de Chile con el número 2104153. Se incluyeron todos los pacientes que se sometieron a AUR entre 2003 y 2019.

Los criterios de selección de los pacientes para someterse a AUR son dolor localizado en el compartimento comprometido, índice de masa corporal inferior a $34 \mathrm{~kg} / \mathrm{m}^{2}$ y alineación reducible en varo o valgo. Los pacientes con dolor difuso de rodilla, mala alineación coronal irreductible moderada o grave, condropatía grado $\geq 3$ o Kellgren y Lawrence $\geq$ III en el compartimento contralateral no son candidatos para AUR. Si el paciente no se queja de dolor en la parte anterior de la rodilla, la condropatía femororrotuliana no es un criterio de exclusión. ${ }^{10}$ Además, la AUR está contraindicada para pacientes con deterioro neuromuscular o inestabilidad de la rodilla, como desgarro del ligamento cruzado anterior, por ejemplo. Mientras tanto, se excluyó a todos los pacientes que se sometieron a ATR o a artroplastia femororrotuliana. La -Figura 1 muestra el diagrama de flujo de la selección de los pacientes.

Se revisaron todas las historias clínicas de los pacientes que se sometieron a una artroplastia de rodilla entre enero de 2003 y mayo de 2019. Los datos extraídos incluyeron fecha de nacimiento, fecha de la cirugía, sexo, tipo de AUR, y el lado en el que se realizó. También se revisaron sucesivos controles médicos por las siguientes complicaciones: reintervenciones, infección superficial, infección profunda, artrofibrosis, necesidad de movilización bajo anestesia, hundimiento tibial, y derivación a unidad de dolor crónico. Antes de la cirugía, todos los pacientes se someten a una radiografía anteroposterior (AP) con carga de peso, una radiografía lateral de la rodilla, una radiografía de Schuss, una radiografía AP de longitud completa de las extremidades inferiores, y una imagen de resonancia magnética (RM) (figura 2).

Para evitar sesgos de selección, se revisó la radiografía postoperatoria en todos los casos para confirmar que el paciente había sido sometido a AUR. Además, el

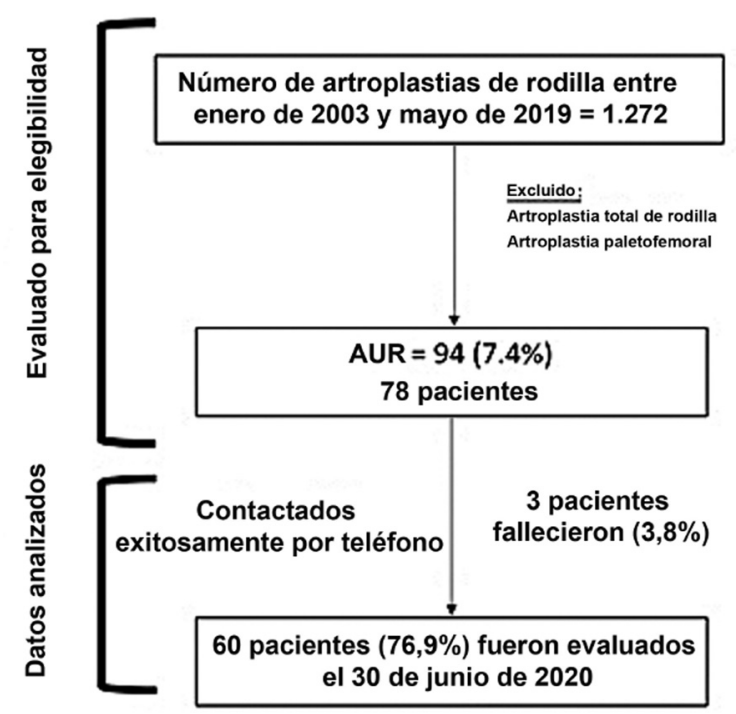

Fig. 1 Diagrama de flujo de la selección de pacientes. procedimiento de AUR fue corroborado durante la entrevista telefónica con los pacientes que fueron contactados con éxito. La historia clínica del paciente fue exhaustivamente revisada para reducir el riesgo de sesgo inherente a un estudio retrospectivo.

Todos los pacientes fueron intervenidos mediante abordaje anterior de rodilla. Se realizó una artrotomía parapatelar medial si era una AUR medial; mientras tanto, se utilizó artrotomía parapatelar lateral en AUR lateral. Solo se utilizaron modelos de rodamiento fijo, siendo ZUK (Zimmer-Biomet, Warsaw, IN, EEUU) y Journey (Smith \& Nephew, Londres, Reino Unido). Primero, el corte tibial se realiza con el objetivo de preservar la mayor cantidad de tibia posible y reproducir la pendiente nativa. Luego, el corte femoral se realiza con el objetivo de restaurar la línea de la articulación y seleccionar el tamaño apropiado del componente femoral (figura 3).

En junio de 2020, un evaluador independiente (DP) se comunicó con todos los pacientes por teléfono. Se aplicó por teléfono una adaptación transcultural validada del índice de artritis de las universidades de Western Ontario y McMaster (WOMAC), ${ }^{11,12}$ un instrumento para evaluar la capacidad funcional de pacientes con osteoartritis de rodilla y cadera, que se validó aún más para evaluar la artroplastia de rodilla. $^{13}$ Por sus propiedades psicométricas, el WOMAC se considera uno de los mejores instrumentos autoinformados en pacientes con artrosis. ${ }^{14}$ Consiste en 3 dimensiones: 5 ítems que evalúan el dolor (puntuación de 0 a 20), 2 ítems de rigidez (puntuación de 0 a 8), y 17 ítems de función física en las actividades diarias (puntuación de 0 a 68). La puntuación es más alta a medida que los pacientes se sienten mal. ${ }^{12} \mathrm{La}$ escala ha sido validada para ser autoadministrada $y$ administrada por teléfono. ${ }^{15}$ Aunque no es la función principal de la escala, las puntuaciones de las 3 dimensiones se pueden sumar y reportar estandarizadas de 0 a $100 .^{14}$

La junta del comité de ética local aprobó este estudio. Todos los pacientes, contactados con éxito por teléfono, dieron su consentimiento verbal para participar en la encuesta utilizando el formulario telefónico aprobado por el comité de ética.

Los datos se resumieron en mediana, rango, y rango intercuartílico (intrquartile range, IQR, en inglés, percentiles 25 a 75). Se comparó la proporción de pacientes contactados con éxito por teléfono con la cohorte total en términos de edad (pacientes mayores de 70 años), sexo, porcentaje de AUR medial, mediana de seguimiento, y proporción de pacientes con más de 5 años de seguimiento. El resultado del WOMAC se comparó respecto a AUR (medial o lateral), edad (mayor o menor de 70 años) y seguimiento (más o menos de 5 años) utilizando la prueba de mediana no paramétrica.

Se utilizó una significancia del 5\%, y se estimó un intervalo de confianza del 95\%. Los datos se procesaron utilizando el programa Stata (StataCorp LLC, College Station, TX, EEUU), versión 15. 


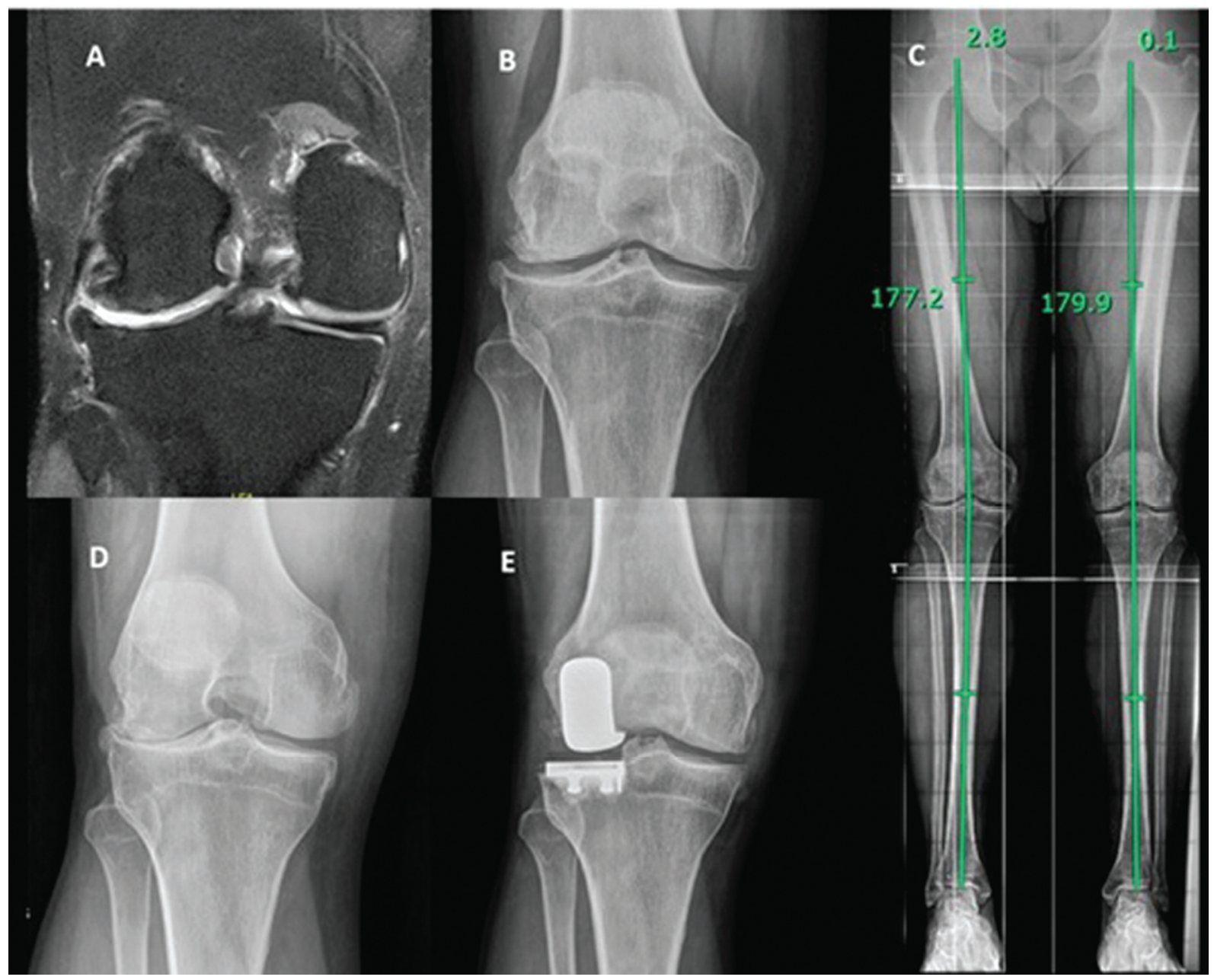

Fig. 2 Estudios de imágenes realizado de forma rutinaria para la selección del paciente. Imagen de resonancia magnética (A) anteroposterior (AP) con carga de peso que muestra una osteoartritis grave del compartimento lateral (B), radiografía AP de longitud completa de miembros inferiores (C), proyección de Schuss (D). Además, se muestra la radiografía AP de rodilla posoperatoria (E).

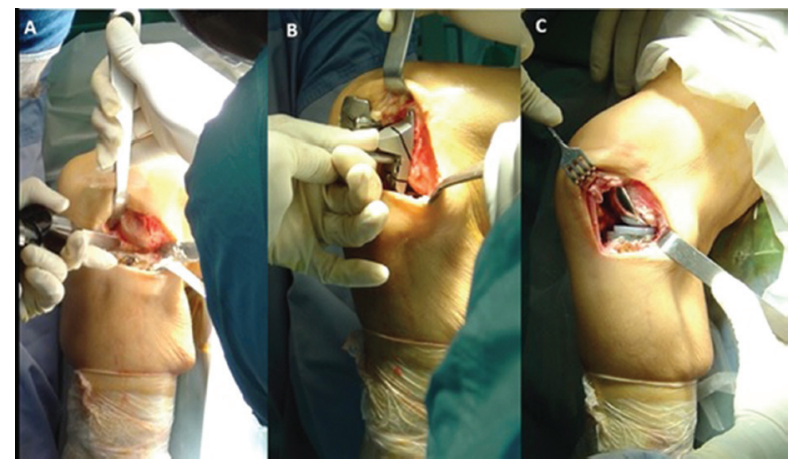

Fig. 3 La AUR lateral se realizó mediante una artrotomía parapatelar lateral. Se muestran el primer corte tibial (A), el corte femoral (B), y la AUR $(C)$.

\section{Resultados}

Se incluyeron 78 pacientes, en un total de 94 AURs; 16 (20,5\%) pacientes fueron sometidos a AUR bilateral. La mediana de edad fue de 64 años (rango: 43 a 85 años; IQR: 58 a 71 añis) y 60 (77\%) pacientes eran mujeres. Del total de AURs, 72 fueron mediales (76,6\%), y 22 (23,4\%) fueron laterales. (-Tabla1). La primera AUR fue en 2003, año en el que se realizó sólo 1 (1,1\%) procedimiento. Además, en 2009 y 2013 sólo se realizó 1 (1,1\%) AUR. La moda se encontró en 2016 , en el que se realizaron un total de 14 (14,9\%) AURs.

En total, $3(3,3 \%)$ pacientes requirieron otra cirugía después de la AUR: 1 paciente requirió revisión para ATR, 1 paciente fue sometido a meniscectomía lateral, y el último necesitó liberación de anserina. La ATR de revisión, para el dolor difuso de rodilla intratable, se realizó después de tres años en otra institución. Entre las complicaciones quirúrgicas, 2 (2,2\%) pacientes tuvieron una infección superficial, y ningún paciente tuvo una infección profunda, requirió movilización bajo anestesia, o requirió una derivación a la unidad de dolor crónico. En total, $1(1,1 \%)$ paciente de 86 años sufrió hundimiento tibial que no requirió revisión. Después de tres meses de tratamiento conservador, el hueso se curó, y el paciente alcanzó un rango de movimiento completo (figura 4). Falleció ocho años después de la cirugía, por enfermedad cardiovascular.

Un total de 60 pacientes (76,9\%), correspondientes a 72 AURs, fueron contactados con éxito por teléfono para el seguimiento final. En el último seguimiento, 3 (3.3\%) pacientes habían 
Tabla 1 Comparación entre la cohorte total y la cohorte contactada con éxito

\begin{tabular}{|l|l|l|}
\hline & Total & $\begin{array}{l}\text { Seguimiento: } \\
30 \text { de junio de } \\
2020\end{array}$ \\
\hline Número de pacientes & 78 & $60(76,9 \%)$ \\
\hline Número AURs & 94 & $72(76,7 \%)$ \\
\hline Edad media* & $64(43$ a 85$)$ & $62(43$ a 85$)$ \\
\hline Más de 70 años $^{*}$ & $25(26,6 \%)$ & $15(25,0 \%)$ \\
\hline Hombres* $^{*}$ & $19(20,2 \%)$ & $11(21,6 \%)$ \\
\hline AUR medial** & $72(76,6 \%)$ & $42(70,0 \%)$ \\
\hline AUR lateral $^{*}$ & $22(23,4 \%)$ & $20(30 \%)$ \\
\hline $\begin{array}{l}\text { Mediana del } \\
\text { seguimiento** }\end{array}$ & $5,0(1,1$ a 16,7$)$ & $4,7(1,1$ a 16,1$)$ \\
\hline $\begin{array}{l}\text { Seguimiento de } \\
5 \text { años o más** }\end{array}$ & $47(50 \%)$ & $35(48,6 \%)$ \\
\hline Revisión de ATR $^{* *}$ & $1(1,1 \%)$ & 0 \\
\hline Subsidencia tibial & $1(1,1 \%)$ & 0 \\
\hline $\begin{array}{l}\text { Necesitaba una } \\
\text { segunda cirugía** }\end{array}$ & $2(2,1 \%)$ & $2(2,8 \%)$ \\
\hline $\begin{array}{l}\text { Infección superficial** } \\
\text { Infección profunda** }\end{array}$ & $2(2,1 \%)$ & $2(2,8 \%)$ \\
\hline
\end{tabular}

Abreviaturas: AUR, artroplastia unicompartimental de rodilla; ATR, artroplastia total de rodilla.

Notas: *Proporción del total de pacientes; ${ }^{* *}$ proporción del total de AURs.

muerto, y $15(19,2 \%)$ no pudieron ser localizados. Las características epidemiológicas se muestran en la tabla 1. La mediana de seguimiento fue de 4,4 años (rango: 1,1 a 16,2 años; IQR: 2,5 a 10,1 años). En el WOMAC, La mediana del dolor fue de 1 (rango: 0 a 12; IQR: 0 a 3); la de la rigidez, de 0 (rango: 0 a 4; IQR: 0 a 0); de la la función física, de 2 (rango: 0 a 29 ; IQR: 0 a 10); y la mediana total fue de 4 (rango: 0 a 44; IQR: 0 a 13) (figura 5).

Los pacientes sometidos a AUR lateral $(n=20,32 \%)$ lograron mejores puntuaciones en el WOMAC total y en todas sus dimensiones que los sometidos a AUR medial $(n=42,68 \%)$. Sólo hubo diferencias estadísticamente significativas en el WOMAC total (prueba de mediana; $p=04329$ ) y en la dimensión de dolor (prueba de mediana; $p=0.0160$ ) (tabla 2).

No se observo diferencias en los puntajes de WOMAC entre pacientes mayores o menores de 70 años (- Figura 6). Los pacientes con un seguimiento de más de 5 años tuvieron una mediana del WOMAC total de 8 (rango: 0 a 26; IQR: 1 a 13). Mientras tanto, los pacientes con menos de 5 años tuvieron una mediana de 1 (rango: 0 a 44; IQR: 0 a 9), y no hubo diferencia estadísticamente significativa (prueba de mediana; $p=0,1329$ ) (figura 7).

\section{Discusión}

Este estudio muestra que los pacientes con AUR tuvieron una proporción alta de resultados buenos/excelentes en el WOMAC. No se encontraron diferencias en el resultado del

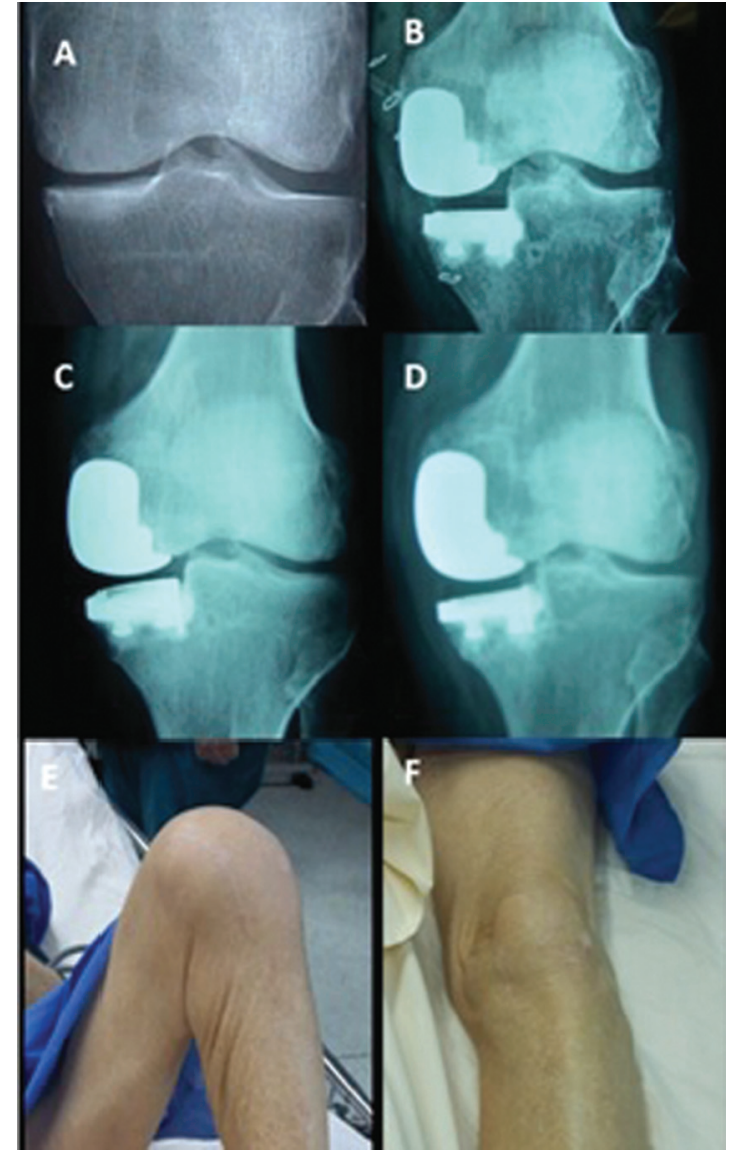

Fig. 4 Subsidencia tibial. Radiografía preoperatoria (A), radiografía AP inmediata (B). Después de tres semanas, se observa una inclinación en la radiografía AP de rodilla (C). La curación ósea se puede ver después de tres meses, sin evidencia de mayor hundimiento o aflojamiento (D). Se muestra el rango de movimiento de la rodilla a los cuatro años de seguimiento (E,F). Fuente: Elaborado por los autores a partir de los resultados del estudio.

WOMAC con respecto a la edad (mayor o menor de 70 años) y al tiempo de seguimiento (más o menos de 5 años). En este estudio, probablemente sólo se encontró $1(1,1 \%)$ revisión de la ATR debido al número de pacientes y al tiempo de seguimiento amplio. El tiempo medio de supervivencia

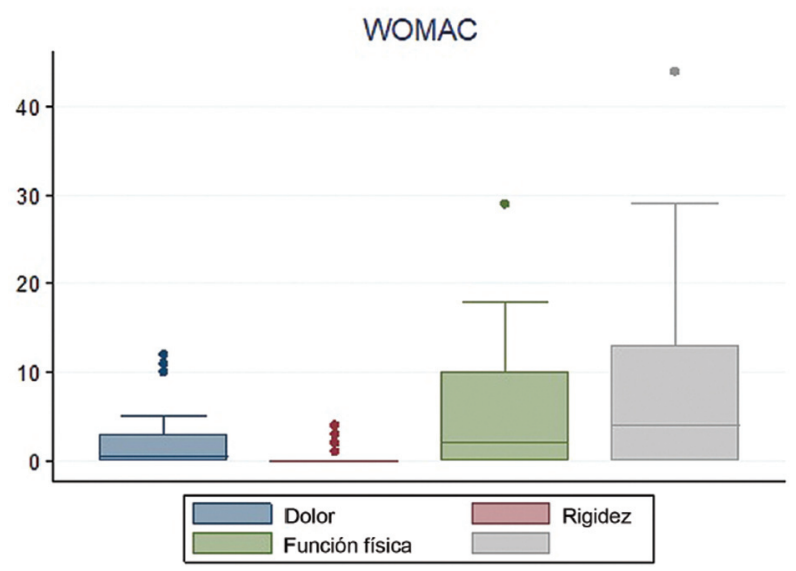

Fig. 5 El índice WOMAC y su distribución de subdimensiones. 
Tabla 2 Comparación del WOMAC entre AUR medial y lateral

\begin{tabular}{|l|l|l|l|}
\hline $\begin{array}{l}\text { Dimensión } \\
\text { del WOMAC }\end{array}$ & AUR medial & AUR lateral & Valor de $\boldsymbol{p}^{*}$ \\
\hline Dolor & $1(0$ a 12$)$ & $0(0$ a 4$)$ & 0.0160 \\
\hline Rigidez & $0(0$ a 4$)$ & $0(0$ a 2$)$ & 0.0640 \\
\hline $\begin{array}{l}\text { Función } \\
\text { física }\end{array}$ & $5(0$ a 29$)$ & $0(0$ a 17$)$ & 0.0566 \\
\hline Total & $6(0$ a 44$)$ & $0(0$ a 21$)$ & 0.0432 \\
\hline
\end{tabular}

Abreviaturas: AUR, artroplastia unicompartimental de rodilla; WOMAC, índice de artritis de las universidades de Western Ontario y McMaster. Nota: *Prueba de mediana no paramétrica.

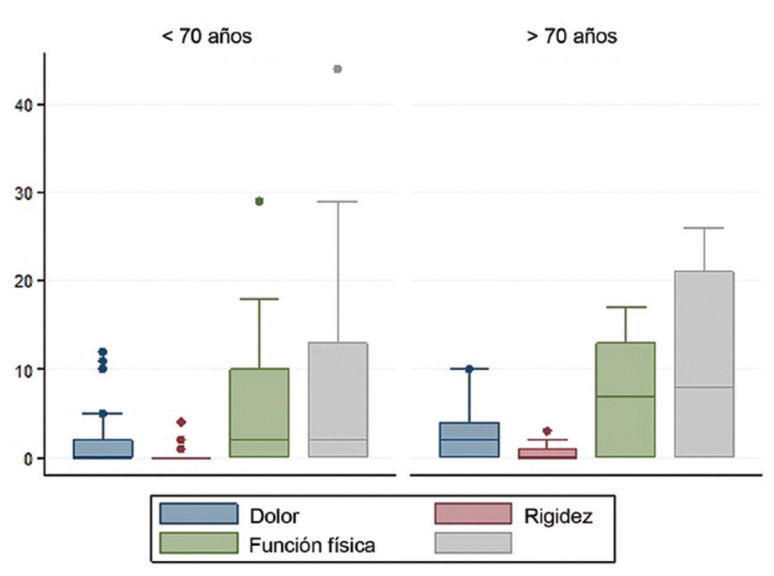

Fig. 6 Distribución de las puntuaciones en el WOMAC entre pacientes mayores o menores de 70 años en el momento de la cirugía.

informado ${ }^{16}$ utilizando la revisión de la ATR como fracaso es del $94,4 \%$ a los 5 años, y del 89,1\% a los 10 años.

Los resultados funcionales de esta cohorte histórica fueron excelentes. Dos cuestiones importantes son fundamentales para obtener buenos resultados en la AUR: la selección y la cantidad de pacientes. ${ }^{10}$ La selección de los pacientes es fundamental para obtener resultados excelentes, especialmente en centros con bajo volumen quirúrgico. ${ }^{17}$ Antoniadis et al. ${ }^{18}$ desarrollaron un novedoso sistema de puntuación preoperatoria para predecir buenos resultados que podría ser útil para identificar a los mejores candidatos para AUR.

La alineación coronal de la extremidad inferior ha sido un tema candente en las indicaciones de AUR. ${ }^{19}$ En nuestro abordaje, el examen físico es obligatorio para establecer si la mala alineación es reducible o fija. En la alineación mecánica, la mala alineación fija entre $5^{\circ}$ y $10^{\circ}$ se considera una indicación relativa. Se debe prestar especial atención cuando se encuentra una mala alineación intraarticular, úes, en la AUR, se ha demostrado consistentemente que se logra una buena satisfacción cuando se restauran la altura y la alineación articular. ${ }^{20,21}$ Mientras tanto, más de $10^{\circ}$ es una contraindicación para AUR. Se han propuesto radiografías de estrés para abordar la reductibilidad de la

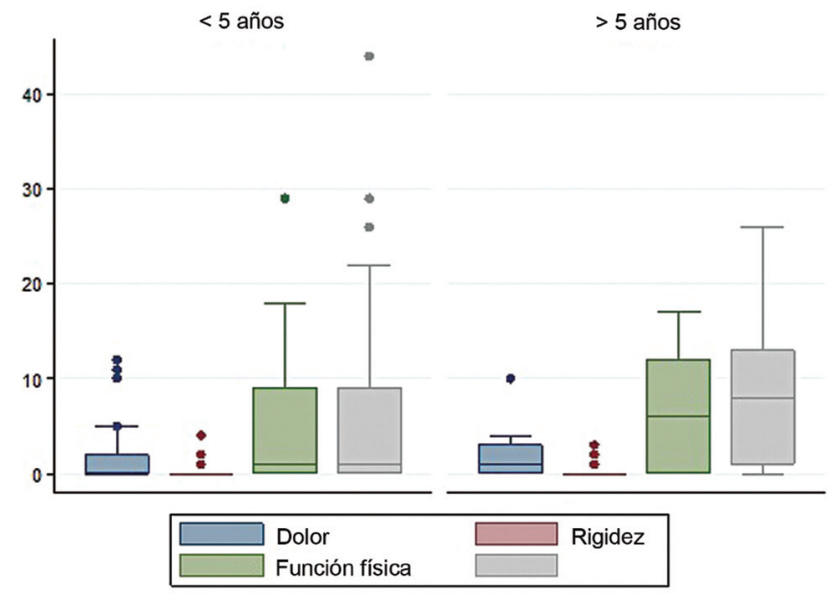

Fig. 7 Distribución de la puntuación en el WOMAC entre pacientes con seguimiento superior o inferior a cinco años.

alineación coronal; sin embargo, no se han reportado hallazgos consistentes. ${ }^{22-24}$

El volumen quirúrgico es crucial para obtener buenos resultados, y nuestra serie muestra que nuestra proporción de pacientes sometidos a artroplastia de rodilla está justo por encima de lo informado en el registro del Reino Unido. ${ }^{5}$ Además, se informa ${ }^{25}$ que doce procedimientos son el umbral para tener una tasa de falla aséptica menor, por lo que nuestro promedio de seis AURs por año es insuficiente. Por lo tanto, pensamos que nuestros buenos resultados están asociados a que el primer autor $(\mathrm{CI})$ estuvo involucrado en todos los casos en los primeros 14 años revisados en esta cohorte.

Los pacientes con osteoartritis de rodilla unicompartimental femorotibial grave tienen tres opciones quirúrgicas: ATR, AUR y osteotomías. Estos tres procedimientos se han utilizado en diferentes tipos de pacientes, pero algunas indicaciones pueden superponerse. $^{26}$ Los estudios de rentabilidad ${ }^{3}$ muestran que la AUR es más eficaz en pacientes mayores de 60 años; mientras tanto, las osteotomías son más útiles en pacientes menores de 60 años. Además, los estudios ${ }^{27,28}$ en pacientes mayores de 75 años muestran que la AUR tuvo resultados funcionales similares, pero tuvo una tasa de complicaciones menor que la de la ATR. En nuestra cohorte, no hubo diferencias estadísticamente significativas en la puntuación en el WOMAC entre los pacientes mayores de 70 años y los pacientes más jóvenes.

Una preocupación importante en el diseño de la artroplastia de rodilla es la capacidad de reproducir la biomecánica natural. Un estudio ${ }^{29}$ de elementos finitos mostró que la AUR es más precisa en la reproducción de la biomecánica nativa de la rodilla que la ATR, incluso si la alineación de la extremidad inferior es leve en varo o valgo. Estos hallazgos se explican porque la AUR preserva los ligamentos cruzados, siendo más anatómicos que en la ATR. Esto es esencial para los pacientes actuales, que son más activos, exigen mejores resultados, y esperan una calidad de vida mejor después de la artroplastia que hace décadas. $^{30}$ Muchos de ellos incluso aspiran a volver a los 
deportes, lo que es consistentemente más probable en pacientes sometidos a AUR que a ATR. ${ }^{4}$

Se ha informado ${ }^{31}$ que la tasa de AUR lateral es del $5 \%$ al $10 \%$ de la tasa de AUR medial, y logra mejores resultados funcionales, pero una tasa de supervivencia más baja. Sin embargo, una revisión sistemática ${ }^{32}$ reciente mostró que las tasas de supervivencia a 5, 20 y 25 años entre AUR medial y lateral son similares. En nuestra cohorte, existe una tendencia significativa a obtener mejores resultados en las AURs laterales, pero esto debe interpretarse con cautela. Los pacientes que requieren AUR medial o lateral tienen un problema de rodilla diferente, por lo que la tendencia encontrada muestra que la AUR lateral es un tratamiento tan bueno como la AUR medial para la osteoartritis unicompartimental. Estos grupos de pacientes tenían diferentes problemas de rodilla, lo que dificultaba la comparación. Existe una apreciación cultural de que realizar la AUR lateral es más complejo; sin embargo, esto está relacionado con que el volumen de cirugías laterales es menor que el de cirugías mediales. Sin embargo, muchos informes $^{33,34}$ muestran que se pueden lograr buenos resultados en el procedimiento lateral, como se informa en este estudio.

Se ha propuesto que la AUR robótica aumenta la orientación y el tamaño del implante, los resultados funcionales, y las tasas de supervivencia. ${ }^{35,36}$ Sin embargo, un metaanálisis reciente ${ }^{37}$ no mostró diferencias en el tiempo medio de supervivencia entre la cirugía asistida por robot y la convencional. Además, los resultados funcionales tampoco fueron significativamente mejores en la AUR asistida por robot. No obstante, esto podría explicarse por el efecto techo de las escalas de resultados funcionales, porque la AUR asistida por robot ha mostrado una precisión sustancialmente mayor en la orientación de los implantes. ${ }^{38,39}$ Probablemente, la cirugía asistida por robot sea el futuro de la AUR. Sin embargo, por ahora, se necesita una mejor comprensión de las orientaciones adecuadas de los implantes, cirujanos capacitados, y un mejor acceso del paciente para mejorar los resultados.

Una limitación de este estudio es que no se trata de una cohorte cautiva. Por lo tanto, los pacientes podrían haber sido revisados a ATR en otro centro. Pero logramos un seguimiento exitoso del 76,9\% de los pacientes, y alcanzando una buena proporción de la cohorte histórica. Además, la única revisión a ATR reportada se realizó en otro centro, que notificó al cirujano $(\mathrm{CI})$, pero no se pudo contactar al paciente en 2020.

\section{Conclusión}

La AUR es un tratamiento eficaz y reproducible para pacientes con osteoartritis femorotibial unicompartimental de rodilla. La edad parece no afectar los resultados funcionales, y la AUR es un tratamiento eficaz en pacientes mayores de 70 años. Estos resultados deberían animar a los cirujanos de rodilla a aprender esta técnica y a los responsables de las políticas de salud pública a considerar la AUR para los pacientes con osteoartritis de rodilla.
Funciones y contribución de los autores

$\mathrm{CI}$ : conceptualización, validación, investigación, curación de datos, redacción (revisión y edición), visualización, supervisión, y administración de proyectos.

MB: metodología, análisis formal, investigación, y redacción (preparación del borrador original, revisión y edición).

DP: metodología, investigación, análisis formal, recursos, curaduría de datos, y redacción (elaboración del borrador original).

CB: investigación, recursos, curación de datos, redacción (revisión y edición), y supervisión.

MP: validación, análisis formal, investigación, recursos, curación de datos, y redacción (preparación del borrador original).

AZ: análisis formal, investigación, redacción (revisión y edición), supervisión, y administración de proyectos.

JH: validación, redacción (revisión y edición), supervisión, y administración de proyectos.

JC: recursos, redacción (revisión y edición), supervisión, y administración de proyectos.

\section{Subvención}

Esta investigación no recibió ninguna subvención específica de agencias de financiamiento en los sectores público, comercial o sin fines de lucro.

\section{Aprobación de ética}

Este estudio fue aprobado por el comité de investigación ética del Hospital Clínico Universidad de Chile, según consta en el certificado número 26, emitido el 26 de junio de 2020.

\section{Disponibilidad de datos}

Los conjuntos de datos utilizados y/o analizados durante el estudio actual están disponibles por medio del autor correspondiente a solicitud razonable.

Conflicto de intereses

Los autores no tienen conflicto de intereses que declarar.

\section{Referencias}

1 Luo TD, Hubbard JB. Arthroplasty Knee Unicompartmental, in StatPearls [Internet]. 2019StatPearls Publishing

2 Marmor L. Marmor modular knee in unicompartmental disease. Minimum four-year follow-up. J Bone Joint Surg Am 1979;61(03): 347-353

3 Smith WB II, Steinberg J, Scholtes S, Mcnamara IR. Medial compartment knee osteoarthritis: age-stratified costeffectiveness of total knee arthroplasty, unicompartmental knee arthroplasty, and high tibial osteotomy. Knee Surg Sports Traumatol Arthrosc 2017;25(03):924-933

4 Witjes S, Gouttebarge V, Kuijer PP, van Geenen RC, Poolman RW, Kerkhoffs GM. Return to sports and physical activity after total and unicondylar knee arthroplasty: a systematic review and meta-analysis. Sports Med 2016;46(02):269-292

5 Willis-Owen CA, Brust K, Alsop H, Miraldo M, Cobb JP. Unicondylar knee arthroplasty in the UK National Health Service: an analysis of candidacy, outcome and cost efficacy. Knee 2009;16(06): 473-478 
6 Vasso M, Antoniadis A, Helmy N. Update on unicompartmental knee arthroplasty: Current indications and failure modes. EFORT Open Rev 2018;3(08):442-448

7 Ingale PA, Hadden WA. A review of mobile bearing unicompartmental knee in patients aged 80 years or older and comparison with younger groups. J Arthroplasty 2013;28(02):262-267.e2

8 Tadros BJ, Dabis J, Twyman R. Short-term outcome of unicompartmental knee arthroplasty in the octogenarian population. Knee Surg Sports Traumatol Arthrosc 2018;26(05): 1571-1576

9 Cook R, Davidson P, White ANIHR Dissemination Centre. Partial knee replacement could be first choice for some patients with osteoarthritis. BMJ 2019;367:15994-15994

10 Kuipers BM, Kollen BJ, Bots PC, et al. Factors associated with reduced early survival in the Oxford phase III medial unicompartment knee replacement. Knee 2010;17(01):48-52

11 Escobar A, Quintana JM, Bilbao A, Azkárate J, Güenaga JI. Validation of the Spanish version of the WOMAC questionnaire for patients with hip or knee osteoarthritis. Western Ontario and McMaster Universities Osteoarthritis Index. Clin Rheumatol 2002;21(06):466-471

12 Roos MK, Lohmander LS. EM, WOMAC Osteoarthritis Index: Reliability, validity, and responsiveness in patients with arthroscopically assessed osteoarthritis. Scand J Rheumatol 1999;28(04):210-215

13 Clement ND, Bardgett M, Weir D, Holland J, Gerrand C, Deehan DJ. What is the minimum clinically important difference for the WOMAC index after TKA? Clin Orthop Relat Res 2018;476(10): 2005-2014

14 Pol EN. Evaluación del paciente y medida de resultados. Aten Primaria 2014;46(Suppl 1):32-38

15 López Alonso SR, Martínez Sánchez CM, Romero Cañadillas AB, Navarro Casado F, González Rojo J. Propiedades métricas del cuestionario WOMAC y de una versión reducida para medir la sintomatología y la discapacidad física. Aten Primaria 2009;41 (11):613-620

16 Bruce DJ, Hassaballa M, Robinson JR, Porteous AJ, Murray JR, Newman JH. Minimum 10-year outcomes of a fixed bearing allpolyethylene unicompartmental knee arthroplasty used to treat medial osteoarthritis. Knee 2020;27(03):1018-1027

17 Hamilton TW, Rizkalla JM, Kontochristos L, et al. The interaction of caseload and usage in determining outcomes of unicompartmental knee arthroplasty: a meta-analysis. J Arthroplasty 2017;32(10):3228-3237.e2

18 Antoniadis A, Dimitriou D, Canciani JP, Helmy N. A novel preoperative scoring system for the indication of unicompartmental knee arthroplasty, as predictor of clinical outcome and satisfaction. Arch Orthop Trauma Surg 2019;139 (01):113-120

19 Deschamps G, Chol C. Fixed-bearing unicompartmental knee arthroplasty. Patients' selection and operative technique. Orthop Traumatol Surg Res 2011;97(06):648-661

20 Chen Y, Fang X, Huang Z, et al. Factors affecting the clinical outcomes including patient satisfaction after Oxford unicompartmental knee arthroplasty: a retrospective study. Arthroplasty 2020;2(01):1-9

21 Kuwashima U, Okazaki K, Tashiro Y, et al. Correction of coronal alignment correlates with reconstruction of joint height in unicompartmental knee arthroplasty. Bone Joint Res 2015;4(08):128-133

22 Kreitz TM, Maltenfort MG, Lonner JH. The valgus stress radiograph does not determine the full extent of correction of deformity prior to medial unicompartmental knee arthroplasty. J Arthroplasty 2015;30(07):1233-1236
23 Waldstein W, Bou Monsef J, Buckup J, Boettner F. The value of valgus stress radiographs in the workup for medial unicompartmental arthritis. Clin Orthop Relat Res 2013;471(12):3998-4003

24 Zhang Q, Yue J, Wang W, Chen Y, Zhao Q, Guo W. FTFA change under valgus stress force radiography is useful for evaluating the correctability of intra-articular varus deformity in UKA. Arch Orthop Trauma Surg 2018;138(07):1003-1009

25 Bini S, Khatod M, Cafri G, Chen Y, Paxton EW. Surgeon, implant and patient variables may explain variability in early revision rates reported for unicompartmental arthroplasty. J Bone Joint Surg Am 2013;95(24):2195-2202

26 Griffin T, Rowden N, Morgan D, Atkinson R, Woodruff P, Maddern G. Unicompartmental knee arthroplasty for the treatment of unicompartmental osteoarthritis: a systematic study. ANZ J Surg 2007;77(04):214-221

27 Fabre-Aubrespy M, Ollivier M, Pesenti S, Parratte S, Argenson JN Unicompartmental knee arthroplasty in patients older than 75 results in better clinical outcomes and similar survivorship compared to total knee arthroplasty. A matched controlled study. J Arthroplasty 2016;31(12):2668-2671

28 Siman H, Kamath AF, Carrillo N, Harmsen WS, Pagnano MW, Sierra RJ. Unicompartmental knee arthroplasty vs total knee arthroplasty for medial compartment arthritis in patients older than 75 years: comparable reoperation, revision, and complication rates. J Arthroplasty 2017;32(06):1792-1797

29 Hopkins AR, New AM, Rodriguez-y-Baena F, Taylor M. Finite element analysis of unicompartmental knee arthroplasty. Med Eng Phys 2010;32(01):14-21

30 Negrín $R$, Duboy J, Iñiguez $M$, et al. Robotic-assisted vs conventional surgery in medial unicompartmental knee arthroplasty: a clinical and radiological study. Knee Surg Relat Res 2021;33(01):5

31 Heyse TJ, Tibesku CO. Lateral unicompartmental knee arthroplasty: a review. Arch Orthop Trauma Surg 2010;130 (12):1539-1548

32 van der List JP, McDonald LS, Pearle AD. Systematic review of medial versus lateral survivorship in unicompartmental knee arthroplasty. Knee 2015;22(06):454-460

33 Fiocchi A, Condello V, Madonna V, Bonomo M, Zorzi C. Medial vs lateral unicompartmental knee arthrroplasty: clinical results. Acta Biomed 2017;88(2S):(Suppl 2):38-44

34 Xue H, Ma T, Wen T, Yang T, Xue L, Tu Y. Predictors of Satisfactory Outcomes With Fixed-Bearing Lateral Unicompartmental Knee Arthroplasty: Up to 7-year Follow-Up. J Arthroplasty 2021;36 (03):910-916

35 Negrín R, et al. Robotic-assisted surgery in medial unicompartmental knee arthroplasty: does it improve the precision of the surgery and its clinical outcomes? Systematic review. J Robot Surg 2020; $\cdots: 1-13$

36 Negrín R, et al. Cirugía Robótica en Artroplastia de Rodilla. Rev Chil Ortoped Traumatol 2019;60(02):67-76

37 Gaudiani MA, Samuel LT, Kamath AF, Courtney PM, Lee GC. Robotic-Assisted versus Manual Unicompartmental Knee Arthroplasty: Contemporary Systematic Review and Metaanalysis of Early Functional Outcomes. J Knee Surg 2020

38 Robinson PG, Clement ND, Hamilton D, Blyth MJG, Haddad FS, Patton JT. A systematic review of robotic-assisted unicompartmental knee arthroplasty: prosthesis design and type should be reported. Bone Joint J 2019;101-B(07):838-847

39 Negrín R, Duboy J, Reyes NO, et al. Robotic-assisted Unicompartmental knee Arthroplasty optimizes joint line restitution better than conventional surgery. J Exp Orthop 2020;7(01):94 